\title{
Limits on First Structure Formation from Pulsar Timing
}

\author{
R. M. Shannon ${ }^{1,2}$ \\ ${ }^{1}$ CSIRO Astronomy and Space Science, Box 76 Epping NSW 1710, Australia \\ email: ryan.shannon@csiro.au \\ ${ }^{2}$ Inernational Centre for Radio Astronomy Research, Curtin University, Bentley, WA 6012, \\ Australia
}

\begin{abstract}
By monitoring the arrival times from millisecond pulsars for years to decades, it is possible to search for, or place limits on, nanohertz frequency gravitational radiation. The most promising source of gravitational waves in this band is a stochastic background emitted from a population of supermassive black hole binaries. As these binaries are the direct product of of galaxy mergers and the properties of the SMBHs correlated strongly with their host galaxies, the gravitational wave emission of the binaries can be used to study how galaxies evolve. Here I discuss how pulsar timing can be used to search for gravitational waves, and how limits on the strength of the background are being used to challenge models of supermassive black hole formation and evolution.
\end{abstract}

Keywords. galaxies:evolution, gravitational waves, pulsars:general

\section{Introduction}

The direct detection of gravitational waves is one of the major goals of physics, and will provide a final confirmation of Einstein's theory of general relativity. These gravitational waves (GWs) also offer great promise for astrophysics as well. GWs are emitted from the most extreme objects in the Universe, many of which are nearly impossible to detect electromagnetically, either because they are too faint to be detected otherwise or hidden obscured by other radiations in their environments. One way to detect GWs uses timing an ensemble of millisecond pulsars referred to as a pulsar-timing array (PTA). I first review how the pulsar timing technique can be used to search for a gravitational wave background (GWB) produced from supermassive black hole binaries. I then present recent limits on the GWB from the Parkes Pulsar Timing Array project, also presented in Shannon et al. (2015). The limit is found to be inconsistent with predictions for the strength of the GWB. I conclude by discussing ways to resolve this conflict and implications for PTA experiments.

\section{Pulsar timing}

Pulsars are rapidly rotating neutron stars that emit beamed radiation above their magnetic axes. If one (or both) of these axes misaligned from the rotation axis but crosses our line-of-sight, the radio emission is observed as pulse. As the neutron stars are rotationally stable, the arrival times of the pulses are regular. In order to make the most precise measurements, it is necessary to observe pulsars at metre and decimetre wavelengths (observing frequencies typically between 0.3 and $3.5 \mathrm{GHz}$ ) with the most sensitive radio telescopes in the world. Signals are detected in cooled receivers, amplified by cryogenic low-noise amplifiers, digitized and then processed by supercomputing backends. 
The fundamental data product currently used in pulsar timing is the pulse time of arrival (TOA). While TOAs can be produced for individual pulses, they are typically formed from the average of many (seconds to hours worth) of pulses to increase the signal to noise ratio of an individual observation and reduce data volume. The measured arrival times are affected by many astrophysical and system dependent processes, Importantly, any process that causes a variation in the path length between the pulsar and the Earth will affect the pulse arrival times. These include both deterministic and stochastic contributions.

Deterministic contributions includes the motion of the Earth around the Sun and the motion of the pulsar about its binary companion, if the pulsar is in a binary system. Deterministic contributions can be directly incorporated into a timing model (Edwards, Hobbs \& Manchester 2006) and effectively removed from the TOAs.

The difference between the TOAs and the model are the residuals, and often contain stochastic noise in excess to the formal TOA uncertainty. Stochastic contributions to TOAs can be divided into two classes: those that are uncorrelated between arrival-time measurements (white noise) and those that are correlated (red noise). Beyond receiver thermal noise, the largest sources of excess white noise can arise from calibration inaccuracies (van Straten 2004) and intrinsic pulse-shape variations (Shannon \& Cordes 2012).

The presence of red noise in pulsar-timing observation is in general more problematic for gravitational-wave detection because it can mimic the signature of the GWs. The most likely source of red noise is associated with intrinsic spin irregularities of the pulsars (Shannon \& Cordes 2010). However, the reflex motion from an ensemble of objects circumpulsar asteroids can also produce a similar red noise similar (Shannon et al. 2013a).

Another source of red noise is associated with the propagation of the radio waves through the warm interstellar medium: the tenuous (density $\lesssim 10^{-2} \mathrm{~cm}^{-3}$ ) warm $\left(10^{4} \mathrm{~K}\right.$ ) ionised plasma that pervades our Galaxy. This plasma induces a frequency-dependent refractive index, and the pulse arrival times are retarded by an amount that is directional proportional to the column density of electrons and the square of the observing frequency. As the pulsar-Earth line of sight varies (due to both the motion of the pulsar in the Galaxy and the motion of the Earth about the Sun), the column density changes.

Stochastic contributions also need to be accounted for, and if possible removed from the TOAs. The effects of dispersion measure variations can be partially subtracted from the residuals (Keith et al. 2013; Lee et al. 2014) if multi-frequency observations are obtained. The other effects need to be included as additional correlated noise (Coles et al. 2011; van Haasteren \& Levin 2013) in the pulsar-timing model.

The most precise arrival times are obtained with millisecond pulsars, which have the highest rotation stability and have have narrow pulses. For this reason MSPs are used to search for GWs.

\section{Gravitational waves and supermassive black hole binaries}

Gravitational waves passing the pulsar-Earth line-of-sight distort space-time and alter the arrival times of pulses, with the Earth and the pulsar acting as test masses, the analogue to the mirrors in an interfereometer. The effects of GWs can be distinguished from other perturbations through studying an ensemble of pulsars (a PTA), because the GWs introduce a quadropolar spatial correlation in the pulse arrival times in the PTA. Other contributions will be uncorrelated between pulsars (e.g., spin-noise), or have monopolar correlation (e.g., variations in terrestrial time, Hobbs et al. 2012) or have 
dipolar correlation (e.g., errors in the solar system ephemeris, Champion et al. 2010)). The correlation is weak because because the effects of the gravitational acting on the pulsars are correlated between pulsars, because the wavelength of the GWs is much shorter than the distance between the Earth and the pulsar, and the pulsar distance is not known to sufficient precision.

Pulsar timing is sensitive to GWs with frequencies between 1 week $^{-1}$ and 1 decade $^{-1}$, i.e, $\approx 3-300 \mathrm{nHz}$. This frequency range makes pulsar timing experiments complementary to existing ground-based and planned space-based laser interferometers, which search for higher frequency GWs.

The most likely source of GWs in the pulsar timing band is associated with merging supermassive black holes, that have grown and evolved along with the Universe. Seed supermassive black holes are formed in the first galaxies. These galaxies merge with other galaxies that also contain black holes. During the merger, the black holes are dragged to the centre of the merged galaxy through dynamical friction and form a bound pair. Dynamical friction (from stars and gas) then causes the supermassive black hole binary to shrink. Eventually, however, GW emission becomes the dominant energy loss, and causes the binary to coalesce quickly. After coalescence, the resultant black hole grows through accretion. Over the age of the Universe, galaxies will experience $\sim 10$ mergers with the galaxies and supermassive black holes hierarchically growing.

The cumulative signal of GWs from all SMBH binaries forms a stochastic GWB that is predicted to be the dominant signal in the pulsar-timing band. The GWB is characterised by a strain spectral index $h_{c}(f)$, which is assumed to have a power-law form

$$
h_{c}(f)=A_{\mathrm{c}, \mathrm{yr}}\left(\frac{f}{1 \mathrm{yr}^{-1}}\right)^{\beta} .
$$

For a GWB produced from binary supermassive black holes evolving by GW emission alone, $\beta=-2 / 3$.

Currently there are three major efforts around the world to detect GWs, based on observations with major telescopes in Australia (Manchester et al. 2013), Europe (Kramer \& Champion 2013), and North America (Demorest et al. 2013). The groups are also working together as part of the International Pulsar Timing Array (IPTA) project (Hobbs et al. 2010).

\section{Placing limits on the gravitational-wave background}

To place a limit on the GWB, we used data from the Parkes pulsar timing array project (Manchester et al. 2013), producing an updated dataset that differed from previous ones analysed from the project (Shannon et al. 2013b). The major difference was that we used only data obtained only in the short-wavelength $(10 \mathrm{~cm})$ band. As a consequence of using only single-band observations, we did not correct for the effects of dispersion measure variations. Longer-wavelength observations were found to be corrupted by excess noise that could not be mitigated (i.e., are not dispersion measure variations). It is presently unclear if the noise is astrophysical (e.g., associated with multi-path propagation in the interstellar medium, Cordes, Shannon \& Stinebring 2015) or is instrumental in nature. In addition to analysing only the $10 \mathrm{~cm}$ data, we also corrected for instrumental offsets between backends. These offsets had previously introduced spurious red noise in the best pulsar in previous analyses (Manchester et al. 2013; Shannon et al. 2013b) and decreased their sensitivity to GWs.

We placed a limit on the GWB using Bayesian methodology (Lentati et al. 2014). We marginalised over the deterministic components of the timing model and searched for 


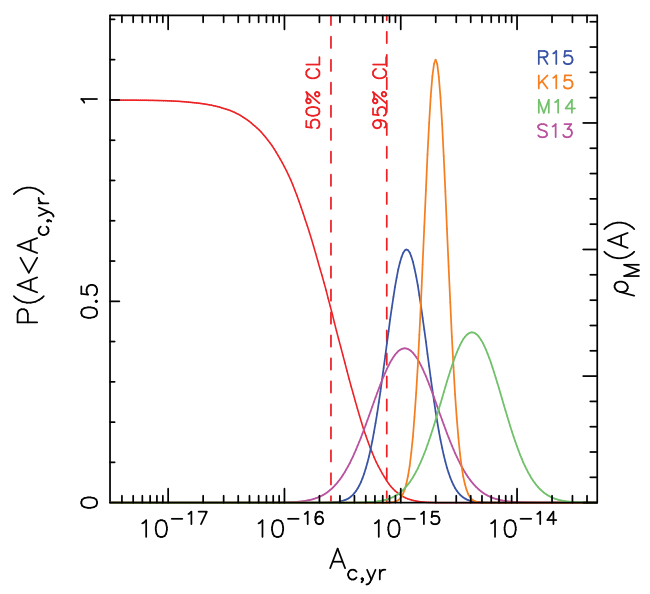

Figure 1. Comparison of limit on $A_{\mathrm{c}, \mathrm{yr}}$ to models. Cumulative posterior distribution for our limit (red curve). We show four recent predictions for the amplitude of the GWB: An emperically derived model (S13, Sesana 2013) is ruled out with 91\% confidence . A model (M14, McWilliams, Ostriker \& Pretorius 2014) that assumes that all grown of SMBH is through merger is ruled out with $99.5 \%$ confidence. A semi-analytic model (K15, Kulier et al. 2015) is excluded with $96 \%$ confidence. A second emperically driven model (R15, Ravi et al. 2015), based on more recent observations of galaxy evolution is ruled out with $94 \%$ confidence.

stochastic contributions to the arrival times, including excess white noise, red noise, and GWs. The methodology allowed use assess the evidence (in the form of probabilities) for the presence (or absence) of the contributions by providing the relative probabilities of the families of models.

We found no evidence for a GWB in our data set. We place a limit of $A_{\mathrm{c}, \mathrm{yr}}=1.0 \times 10^{-15}$ with $95 \%$ confidence (Shannon et al. 2015). The cumulative distribution function (CDF) for $A$, as inferred from our data is shown as the red curve in Figure 1. Because the sensitivity of PTA data sets are $\propto A^{2}$, this limit is factor of 5 improvement over the previously best published limit, $A_{c, y r}=2.4 \times 10^{-15}$ (Shannon et al. 2013b). For the best pulsar, PSR J1909-3744, we find no evidence for red noise in the data set. For the second-best pulsar, PSR J1713+0747, we find evidence for shallow red noise in the data set. This red noise could be associated For the third-best pulsar, PSR J0437-4715, we find evidence for strong red noise that is inconsistent in amplitude with the limit on red noise in the best pulsar. The fourth best pulsar, PSR J1744-1134, shows no evidence for red noise, but also does not affect the combined limit, so demarcates the boundary between important and unimportant pulsars.

In the PPTA sample (and indeed all current timing array samples, Arzoumanian et al. 2014) the individual pulsars have varying levels of sensitivity to the GWB, with the most precisely timed pulsars having orders of magnitude greater sensitivity than the worst pulsars, so an unbiased limit can be produced using only observations of four pulsars.

\section{Implications for the formation and evolution of galaxies}

We can directly compare predictions for $A$ to the models and calculate the probability that the models are consistent with our limit. This probability is the integral of the CDF of the limit and the probability density functions of the model (Shannon et al. 2013b). In Figure 1, we show the comparison of the CDF of the limit to the latest predictions for the value of $A$. We find that all models are in disagreement with the limit, with models that 
predict the strongest GWB ruled out with $>99.5 \%$ confidence (McWilliams, Ostriker \& Pretorius 2014), and all models inconsistent with $>91 \%$ probability.

There are a number of explanations for the low limit on the GWB. Firstly, it is possible that only a small fraction of the first galaxies contain seed black holes that, through merger and accretion, grow into the SMBHs in the lower-redshift Universe. For example, the quasar population at a redshift of $z \sim 2$ is sufficiently unconstrained to allow for much lower seed fractions of black holes (and lower occupations at $z \lesssim 1$ where the bulk SMBH binaries contribution to the GWB occur). In this case, after many galaxy mergers, SMBH binaries would not be produced, and the GWB amplitude would be lower, but still have the power-law form.

It is also possible that galaxy mergers are rarer than currently estimated. In this case, the SMBH merger rate (and the GWB amplitude) would be lower than currently predicted. However, the galaxy merger rate at a redshift $z \lesssim 1$, where the bulk of the detectable signal originates, is thought to be on strong observational footing.

It is also possible that the time-scale for SMBHs to form a binary is much longer than currently predicted. In this case, fewer SMBH binaries would not be produced in a Hubble time and again the GWB amplitude would be lower than predicted. However, widely separated quasars and AGN are uncommon, suggesting that dynamical friction brings SMBH into the centres of galaxies. Additionally, many mechanisms have been identified theoretically to cause SMBHs to be dragged to galaxy centres and form a gravitationally bound pair.

It is also possible that after forming a binary, orbit shrinking (necessary to get the binary to sub-parsec separation the stage of emitting GWs) is too slow. This final-parsec problem has been well studied theoretically. Many mechanisms have been proposed to solve this problem by using a combination of friction from stars, gas, and aspherical gravitational potentials to cause SMBH binaries to in-spiral. However observations, now including ours, could imply that orbital shrinking stalls and binaries do not enter the GW-bright stage within the age of the Universe.

Conversely, it is possible that the environments surrounding SMBH binaries are sufficiently dense that they (and not GWs) can drive in-spiral through the pulsar timing band. These dense environments (stars, gas, or both) are required to solve the final-parsec problem. However, if sufficiently dense drag from gas or dynamic friction from stars can drive inspiral when the $\mathrm{SMBH}$ binaries should be emitting $\mathrm{nHz}$ frequency GWs. In this case, fewer GWs would be emitted at lower frequencies, and the GWB amplitude would be expected to have a break in the power-law strain spectrum. In this case, it is possible that the GWB does not have a power-law strain spectra, but has a break or turnover at low frequencies, where PTAs have their greatest sensitivity.

\section{Implications for gravitational-wave detection by PTAs}

Our result suggests that revised observing strategies could increase the sensitivity of PTAs to GWs.

Interestingly, we obtain our highest timing precision at a relatively short wavelength $(10 \mathrm{~cm})$ for pulsar timing. Our longer-wavelength observations appear to be corrupted by excess noise. It is unclear if this noise is associated with instrument-specific problems (insufficient calibration or radio-frequency interference) or is astrophysical in origin. If the noise is astrophysical, we would expect that the noise would be present in other PTA observations, and the PTAs should consider increasing the fractional time spent observing at short wavelengths. And that short-wavelength capabilities should be included in 
the design of next-generation radio telescopes such as Five Hundred Metre Aperture Telescope (Hobbs et al. 2014) and the Square-Kilometre Array (Janssen et al. 2015).

Improved limits will also be realised when data from all the major pulsar timing array projects are combined, as part of the International Pulsar Timing Array (IPTA) project (Hobbs et al. 2010). These observations will also enable us understand the nature of the excess low-frequency noise and determine whether it is common to all telescopes (hinting towards an astrophysical origin) or is only seen in a few pulsars. In the shortterm, ultra wide band instrumentation (capable of observing over a 6:1 frequency range) is being developed that will allow us to be better identify systematic origins for the low-frequency noise and better correct for dispersion measure variations.

Given the disparity in sensitivity between the highest precision timing pulsars and the rest, it is likely that the first evidence for GWs will be evidence for excess red noise in the best pulsar, J1909-3744. If the GWB is diminished at low frequencies because of additional environmental effects, higher cadence observing campaigns that target higher frequency GWs would be beneficial. PTA observations need to be optimised to detect a weaker than expected GWB.

Even though we have not detected the GWB, our results show that the relevance of PTAs for studies of galaxies and supermassive black holes. Until a detection is made, improved limits on the GWB will continue to provide important, unique constraints on galaxy formation and evolution.

\section{Acknowledgement}

The Parkes radio telescope is part of the Australia Telescope, which is funded by the Commonwealth of Australia for operation as a National Facility managed by the Commonwealth Scientific and Industrial Research Organisation (CSIRO).

\section{References}

Arzoumanian, Z., et al.: The NANOGrav Collaboration 2014, ApJ, 794, 141

Champion, D. J., Hobbs, G. B., Manchester, R. N., et al. 2010, ApJ, 720, L201

Coles, W., Hobbs, G., Champion, D. J., Manchester, R. N., \& Verbiest, J. P. W., 2011, MNRAS, 418,561

Cordes, J. M., Shannon, R. M., \& Stinebring, D. R. 2015, ApJ, submitted; arXiv:1503.08491

Demorest, P. B., et al. 2013, ApJ, 762, 94

Edwards, R. T., Hobbs, G. B., \& Manchester, R. N. 2006, MNRAS, 372, 1549

Hobbs, G., et al. 2010, Classical Quant. Grav., 27, 084013

Hobbs, G., Coles, W., Manchester, R. N., et al. 2012, MNRAS, 427, 2780

Hobbs, G., Dai, S., Manchester, R. N., Shannon, R. M., Kerr, M., Lee, K. J., \& Xu, R. 2014, Res. Astron. Astrophys., in press; arXiv:1407.0435

Janssen, G., Hobbs, G., McLaughlin, M., et al. 2015, "Gravitational Wave Astronomy with the SKA," in Advancing Astrophysics with the Square Kilometre Array, Proc. Sci., eds. T. L. Bourke, et al., id 37

Keith, M. J., Coles, W., Shannon, R. M., et al. 2013, MNRAS, 429, 2161

Kramer, M. \& Champion, D. J. 2013, Classical Quant. Grav., 30, 224009

Kulier, A., Ostriker, J. P., Natarajan, P., Lackner, C. N., \& Cen, R. 2015, ApJ, 799, 178

Lee, K. J., Bassa, C. G., Janssen, G. H., et al. 2014, MNRAS, 441, 2831

Lentati, L., Alexander, P., Hobson, M. P., et al. 2014, MNRAS, 437, 3004

Manchester, R. N., Hobbs, G., Bailes, M., et al. 2013, PASA, 30, 17

McWilliams, S. T., Ostriker, J. P., \& Pretorius, F. 2014, ApJ, 789, 156

Ravi, V., Wyithe, J. S. B., Shannon, R. M., \& Hobbs, G. 2015, MNRAS, 447, 2772

Sesana, A. 2013, MNRAS, 433, L1

Shannon, R. M. \& Cordes, J. M. 2010, ApJ, 725, 1607 
Shannon, R. M. \& Cordes, J. M. 2012, ApJ, 761, 64

Shannon, R. M., Cordes, J. M., Metcalfe, T. S., et al. 2013a, ApJ, 766, 5

Shannon, R. M., Ravi, V., Coles, W. A., et al. 2013b, Science, 342, 334

Shannon, R. M., Ravi, V., Lentati, L. T., et al. 2015, Science, 349, 1522

van Haasteren, R., Levin, Y. 2013, MNRAS, 428, 1147

van Straten, W. 2004, ApJS, 152, 129 